\title{
Fibroma Cemento Osificante: Análisis Clínico, Radiológico e Histológico de 2 Casos en una Misma Familia
}

\author{
Cemento-Ossifying Fibroma: Clinical, Radiological and \\ Histological Analysis of 2 Cases in One Family
}

Ilich Vargas F.",*; Cristopher Mayer O.."; Percival Hervozo S.* \& Eduardo Navia G. ${ }^{* * *}$

VARGAS, F. I.; MAYER, O. C.; HERVOZO, S. P. \& NAVIA, G. E. Fibroma cemento osificante: análisis clínico, radiológico e histológico de 2 casos en una misma familia. Int. J. Odontostomat., 5(3):270-278, 2011.

RESUMEN: El Fibroma Cemento Osificante (FCO) es una lesión pseudo tumoral benigna de origen odontogénico, que se ubica preferentemente a nivel de molares y premolares mandibulares. Nuestro objetivo es presentar casos de FCO dentro de una misma familia, describiendo las características clínicas, radiográficas, histológicas, tratamiento y seguimiento de cada uno de ellos. Dos pacientes hermanos con FCO mandibulares atendidos en el Hospital de Carabineros fueron incluidos en este estudio. La evolución completa de cada caso es expuesta, resaltando el componente familiar de esta patología poco prevalente. Todos los pacientes fueron tratados con remoción completa de la lesión y relleno del defecto óseo con injerto autólogo de cresta iliaca o con injerto óseo particulado sintético (ChronOS $($ ). Un paciente presento recidiva de la lesión y posteriormente un nuevo FCO en el lado contra lateral. En este tipo de lesiones, es muy importante valerse de todas las herramientas diagnosticas que estén a nuestro alcance, ya que la baja ocurrencia de estas lesiones, junto con la gran similitud tanto clínica, radiográfica e histológica que presenta con otras entidades patológicas de los maxilares, hacen que un estudio superficial, puedan llevar a un diagnostico y tratamiento erróneo, afectando la calidad de vida del paciente. El tratamiento de estas lesiones es conservador, y las recidivas son raras.

PALABRAS CLAVE: fibroma, neoplasia benigna, injerto óseo, diagnostico diferencial.

\section{INTRODUCCIÓN}

Los tumores osteofibrosos de cabeza y cuello fueron descritos por primera vez como una entidad patológica hace ya más de 60 años (Domínguez \& Martín-Granizo, 2004). El Fibroma Cemento Osificante (FCO) es una neoplasia benigna de origen mesodérmico, específicamente de células multipotenciales del ligamento periodontal (Canger et al., 2004; Smith et al., 2009).

EI FCO se considera una neoplasia osteogénica benigna, bien delimitada, y muy pocas veces encapsulada, la cual consiste en tejido fibroso con cantidades variables de material mineralizado similar al tejido óseo y/o cemento (Lombardi et al., 2009). Los FCO no presentan una etiología clara, pero han sido relacionados con procesos inflamatorios como la periodontitis, exodoncias y antecedentes traumáticos (Domínguez \& Martín-Granizo, 2004; Canger et al., 2004).
El FCO es una lesión rara, correspondiendo al $0,1 \%$ del total de los tumores odontogénicos (Gunaseelan et al., 2007), y que típicamente afecta más a mujeres $(5: 1)$ entre la $3^{a}$ y $4^{\text {a }}$ década de vida, y que tiene mayor incidencia en el área molar y premolar mandibular, aunque también se puede encontrar en maxilar, hueso cigomático, etmoides, frontal, región orbitaria y otros (Domínguez \& Martín-Granizo; Lombardi et al., 2009; Pérez-García et al., 2004; Young \& Rowson, 2006; Guzmán \& Hernández, 2008).

Clínicamente los FCO son asintomáticos, por lo cual generalmente son hallazgos radiológicos, aunque en casos de larga evolución pueden originar una asimetría facial creciente debido a un abombamiento de la cortical ósea. A pesar de ser una lesión intraosea, se han descrito casos que afectan encía y tejidos blandos

- Cirujano Maxilofacial Hospital Mutual de Seguridad, Santiago, Chile.

* Cirujano Maxilofacial Hospital de Carabineros, Santiago, Chile.

${ }^{* * *}$ Cirujano Dentista, residente Cirugía Maxilofacial Hospital Mutual de Seguridad, Santiago, Chile. 
donde se puede observar un tejido ulcerado, doloroso, friable y sangrante al tacto (Manganaro et al., 1997).

La apariencia radiológica es muy variable dependiendo de la etapa de su desarrollo y de su tamaño (Domínguez \& Martín-Granizo). Es así que en estados más tempranos se presentan como áreas radiolúcidas uni o multiloculares bien delimitadas, y a medida que maduran y crecen se observan como zonas radiolúcidas bien delimitadas con distintos grados de radiopacidad (Lombardi et al.). Es muy raro que estas lesiones provoquen desplazamiento o reabsorción radicular, pero puede darse sobre todo en lesiones de mayor tamaño o de larga evolución.

Histológicamente, los FCO se caracterizan por presentar un estroma fibroso con células fusiformes entremezcladas con tejido calcificado, vascularización moderada, y muy poca actividad mitótica y atipias celulares (Bertolini et al., 2002). Es posible observar células gigantes e infiltrado inflamatorio agudo con PMNN (Kaplan et al., 2007). Algunos autores señalan que la lesión se encuentra rodeada de una capsula fibrosa, lo cual es muy importante en el diagnostico diferencial con la displasia fibrosa (Su et al., 1997), sin embargo, otros señalan que este tejido no corresponde a una capsula si no que a una transición entre tejido displásico y hueso sano (Lello \& Sparro, 1985).

El FCO presenta un crecimiento lento pero constante, por lo cual se requiere la extirpación completa de la lesión. Los rangos de recurrencia varían entre un 0 a $28 \%$ cuando la lesión es tratada mediante enucleación (Pérez-García et al., 2004). Es fundamental un seguimiento radiográfico y clínico a largo plazo. La resección en bloque podría estar indicada en casos que recidivan después de la eliminación primaria con curetaje quirúrgico, o en casos que la lesión sea extensa (Sakode et al., 1992). No existe evidencia de que el FCO pueda transformarse en una lesión maligna.

Es necesario una minuciosa historia clínica y un análisis radiológico detallado, junto con al estudio anatomo patológico (previa biopsia representativa de la lesión) para así realizar un adecuado diagnostico diferencial. Dentro de las lesiones fibro óseas a descartar se encuentran: Displasia fibrosa, Displasia cemento ósea focal, FCO juvenil, Osteoma osteoide, Osteoblastoma, Cementoblastoma benigno, Fibro odontoma ameloblástico, Cementoblastoma, Osteomielitis y Osteosarcoma (Domínguez \& MartínGranizo; Lombardi et al.).
El objetivo de este trabajo es presentar tres casos de fibromas cemento osificantes mandibulares ocurridos en dos pacientes de una misma familia, sus características clínicas, radiográficas e histológicas, además de su abordaje terapéutico.

\section{REPORTE DE CASOS}

CASO 1. Paciente sexo masculino, de 27 años de edad, sin antecedentes mórbidos de relevancia, es referido al Hospital de Carabineros de Chile por un aumento de volumen a nivel de cuerpo mandibular de lado derecho, de 6 meses de evolución, asintomático. El paciente relata haber sufrido perdida de piezas 4.7, 4.6 y 4.5 hace 5 años. Al examen extraoral es posible observar aumento de volumen discreto en región mandibular del lado derecho (Fig. 1). Al examen intraoral se observa el aumento de volumen, de consistencia dura en relación a reborde desdentado, con expansión hacia vestibular y lingual, con una mucosa de recubrimiento de aspecto normal (Fig. 2). El paciente relata hipoestesia labial inferior.

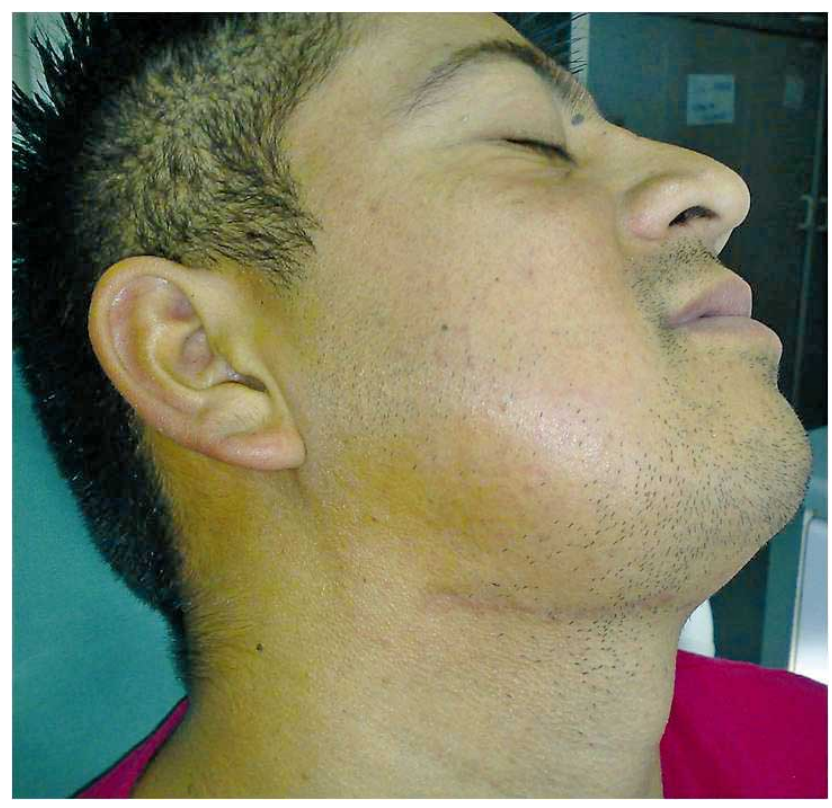

Fig. 1. Examen extraoral se observa aumento de volumen en región mandibular derecha.

Tanto en la radiografía panorámica como en el TC es posible apreciar una lesión expansiva, de densidad mixta y límites netos, con halo radiolúcido y corticalizado. El canal mandibular se observa desplazado hacia borde basilar (Figs. 3 y 4 ). 


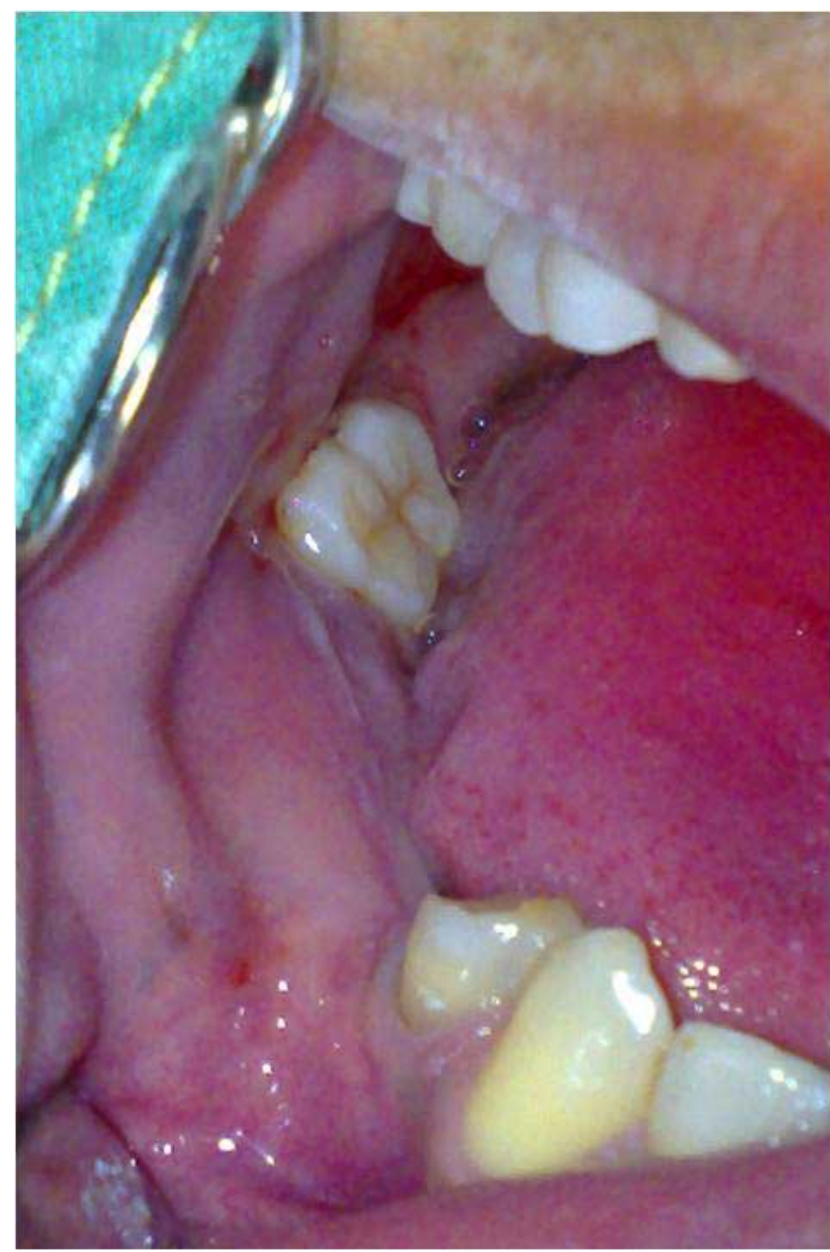

Fig. 2. Examen intraoral se observa aumento de volumen.

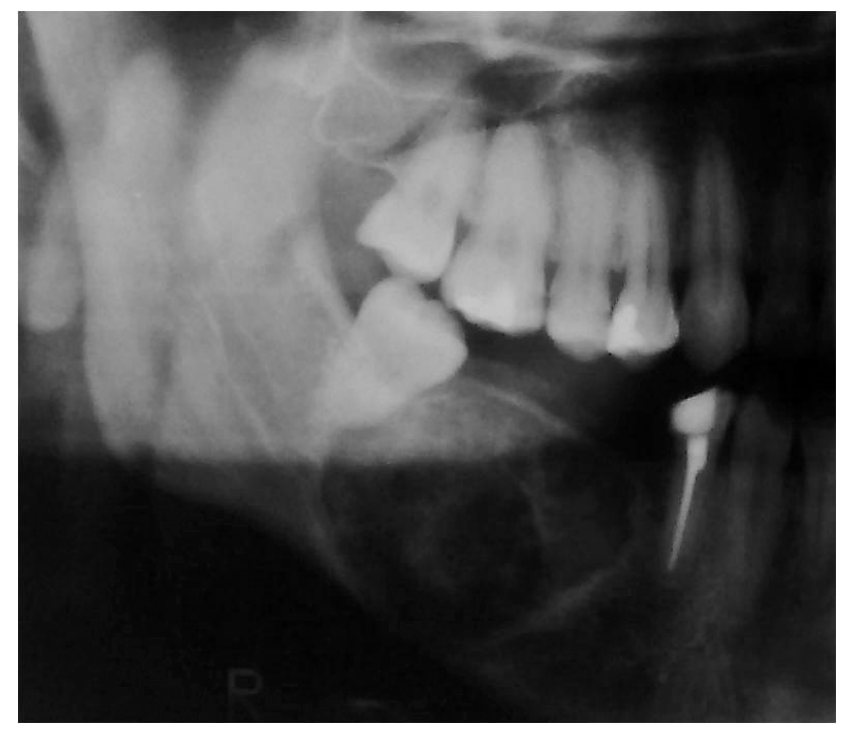

Fig. 3. Radiografía panorámica se aprecia lesión expansiva, de densidad mixta y límites netos, con halo radiolúcido y corticalizado.

Se realizó biopsia incisional bajo anestesia local, en la cual se obtiene un tejido de consistencia dura, que posteriormente es enviado a estudio histopatológico, donde es posible observar tejido fibroso con una densa población fusocelular, sin atipias, los cuales rodean múltiples depósitos de material amorfo eosinófilo, parcialmente calcificados. Tejido óseo laminillar con actividad osteoblástica reactiva. En pabellón central bajo anestesia general mediante abor-

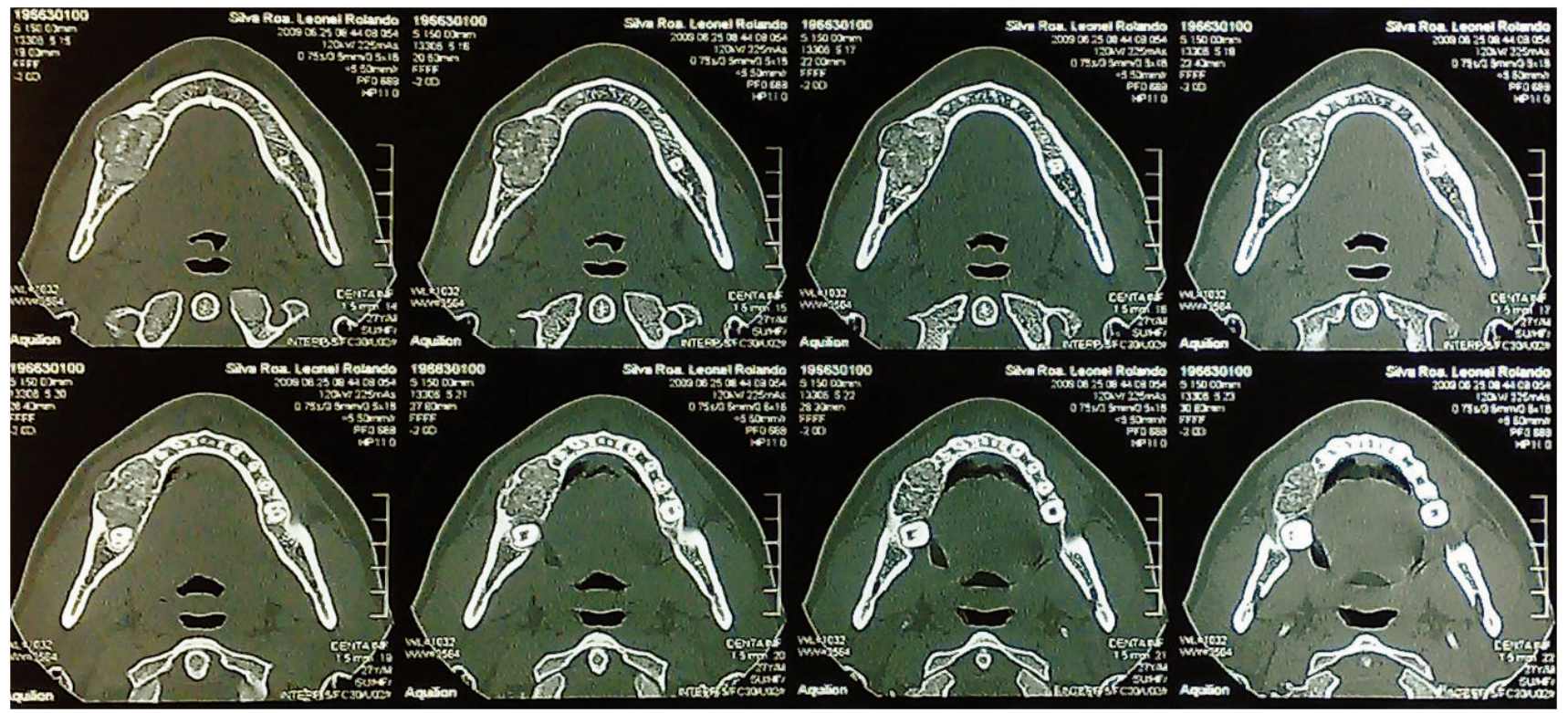

Fig. 4. TC se aprecia lesión expansiva, de densidad mixta y límites netos, con halo radiolúcido y corticalizado. El canal mandibular se observa desplazado hacia margen basilar. 
daje submandibular se realiza la resección completa de lesión más curetaje y reconstrucción inmediata del defecto con injerto autólogo de cresta iliaca (Figs. 5 y 6). El informe histopatológico confirma el diagnostico de fibroma cemento osificante mandibular.

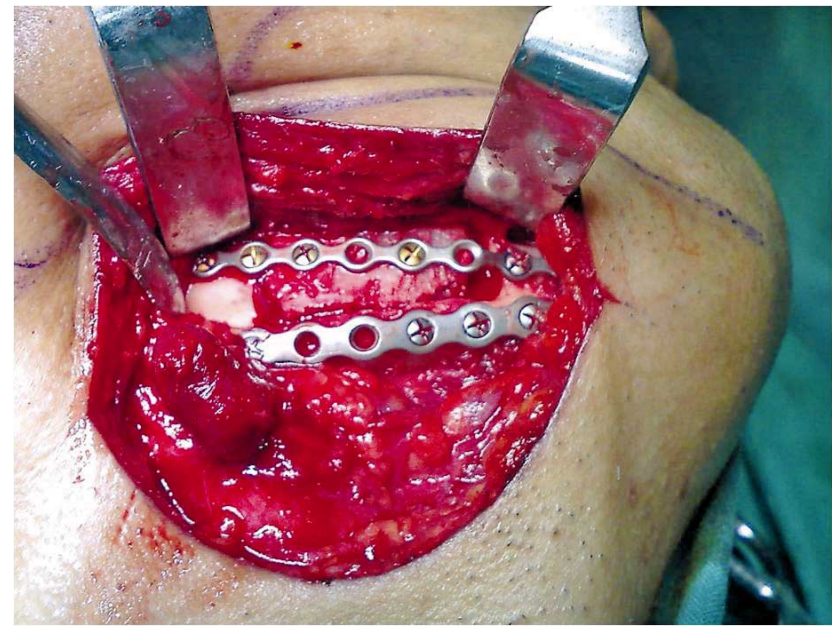

Fig. 5. Resección completa de lesión más curetaje y reconstrucción inmediata del defecto con injerto autólogo de cresta iliaca.

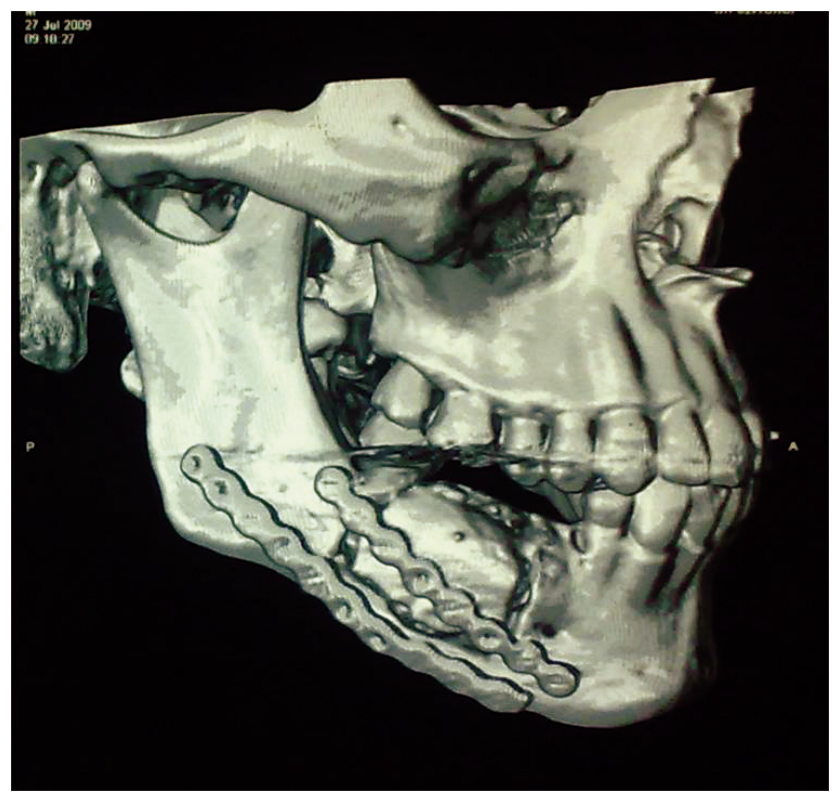

Fig. 6. TC mostrando reconstrucción inmediata del defecto con injerto autólogo de cresta iliaca.

Durante el post operatorio el paciente evoluciona favorablemente, con dolor moderado en sitio dador de injerto. Se instaura tratamiento kinesiológico en base a drenaje linfático, electro estimulación y ejercicios para recuperar movilidad del labio inferior, y se mantiene en controles clínicos. Al segundo mes de contro- les el paciente se presenta con salida de secreción en sitio operatorio, por lo cual se realizan lavados periódicos con Clorhexidina al 0,12\% y tratamiento antibiótico. Finalmente el injerto se expone por lo cual se programa realizar un aseo quirúrgico y retiro de injerto libre bajo anestesia general.

A los 4 meses post aseo quirúrgico el paciente acude con un nuevo aumento de volumen en sitio operatorio, además de un nuevo aumento de volumen vestibular desde pieza 3.2 a 3.6, de consistencia dura y mucosa de aspecto normal (Fig. 7). Se realiza TC donde se confirma la recidiva de la lesión y la nueva lesión radiolúcida en el lado contralateral (Figs. 8 y 9). Bajo anestesia local se realiza biopsia incisional de la nueva lesión que arroja como resultado un nuevo fibroma cemento osificante.

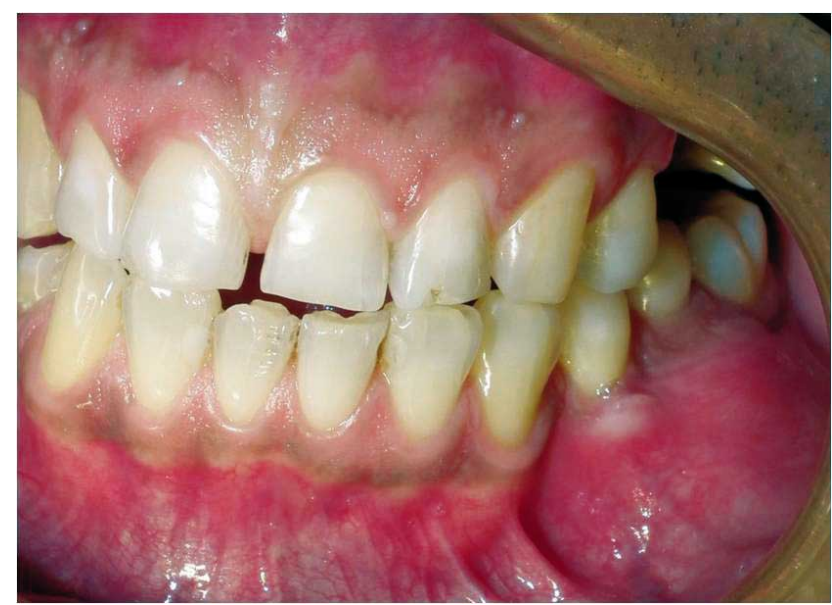

Fig. 7. 4 meses post aseo quirúrgico se observa un nuevo aumento de volumen en sitio operatorio.

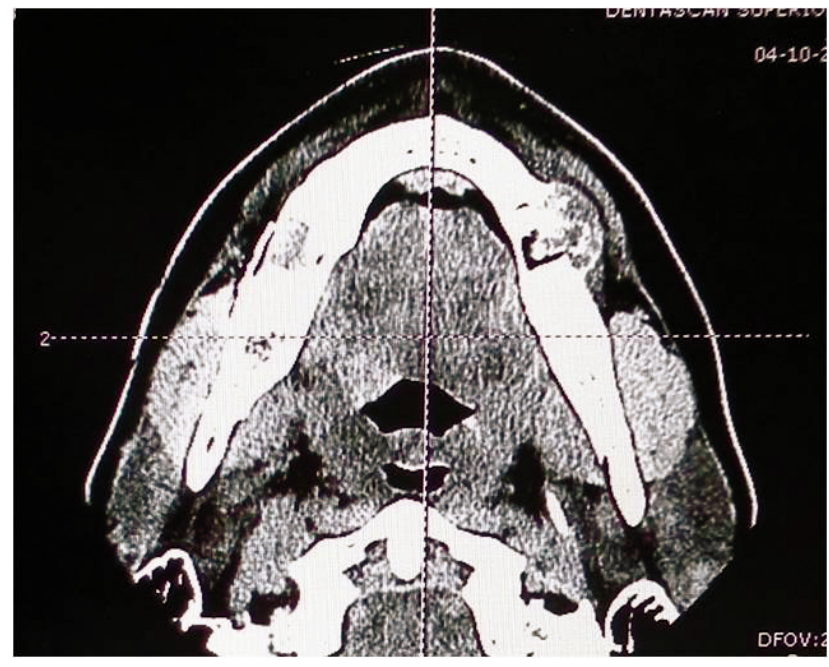

Fig. 8.TC se observa la recidiva de la lesión y la nueva lesión radiolúcida en el lado contralateral. 


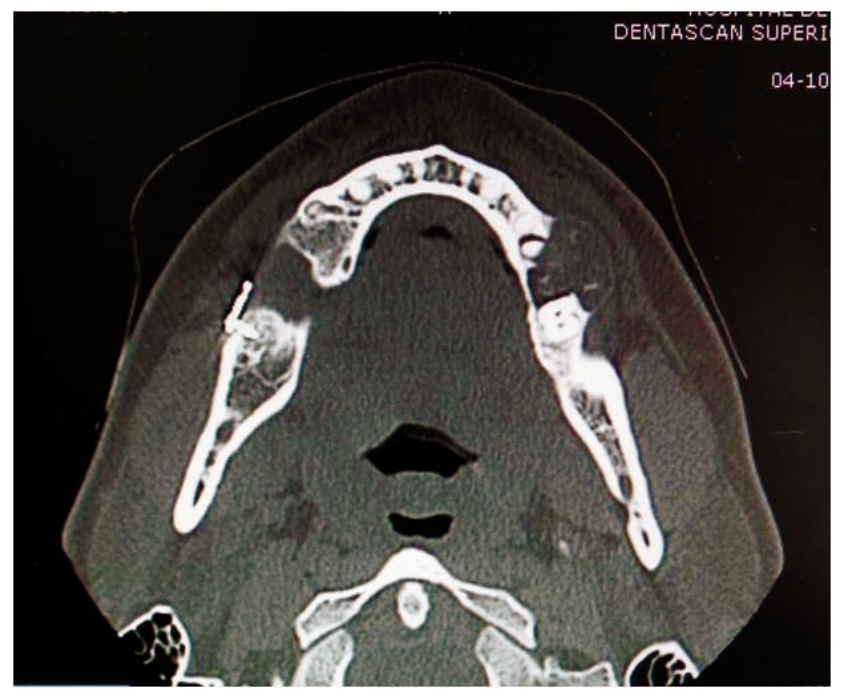

Fig. 9.TC recidiva de la lesión y nueva lesión radiolúcida en el lado contralateral.

En pabellón, bajo anestesia general se realiza la resección completa de la lesión más las piezas dentarias afectadas junto al legrado óseo (Figs. 10 y 11). En cavidad operatoria se instala injerto óseo aloplástico en partículas ChronOs (1,0cc), más gelita. El informe histopatológico final confirma el diagnostico de fibroma cemento osificante mandibular.

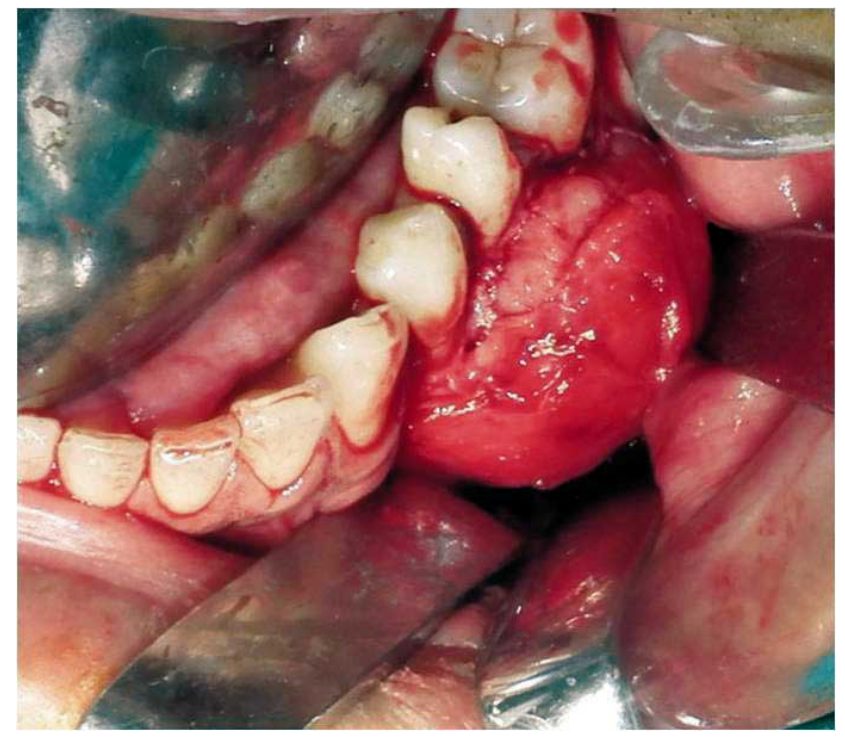

Fig. 10. Resección completa de la lesión más las piezas dentarias afectadas junto al legrado óseo.

CASO 2. Cinco meses después, una paciente de sexo femenino, de 28 años de edad, hermana del paciente del caso numero 1 ingresa a Hospital de Carabineros de Chile por un aumento de volumen mandibular

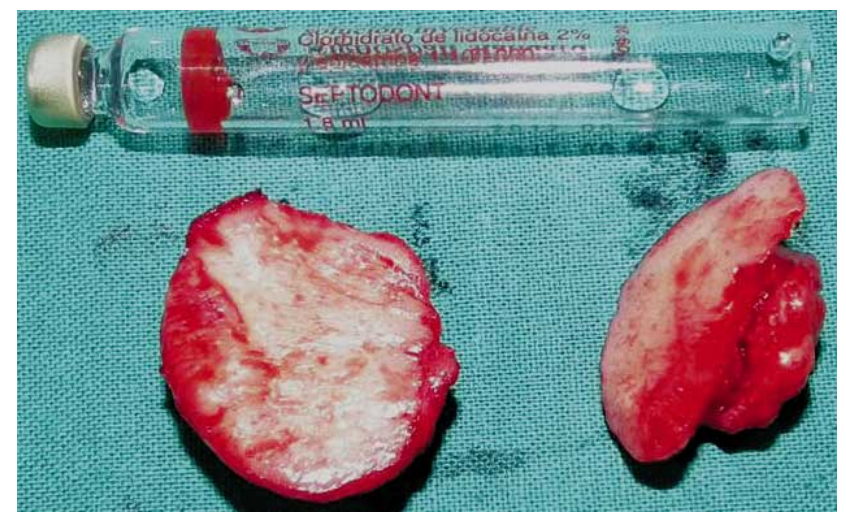

Fig. 11. Piezas dentarias afectadas junto al legrado óseo.

asintomático a nivel de cuerpo lado izquierdo de 4 meses de evolución. Al examen intraoral, se observa un aumento de volumen duro, firme, no fluctuante por vestibular a nivel de piezas 3.5 y 3.6. La mucosa de recubrimiento presenta un aspecto normal (Fig. 12). No existe movilidad dentaria de los dientes afectados.

En la radiografía panorámica se puede observar una imagen radiolúcida interradicular y periapical

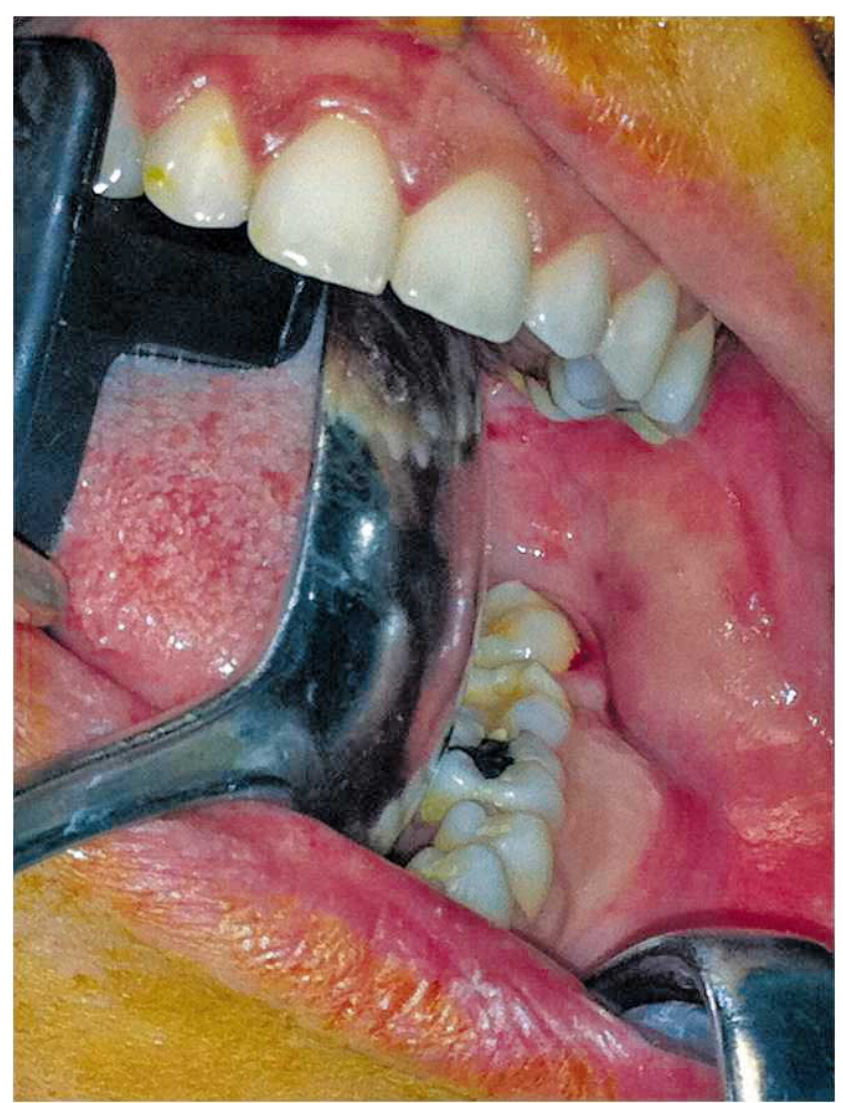

Fig. 12. Mucosa de recubrimiento de aspecto normal. 
corticalizada con zonas radiopacas, de aspecto multilocular a nivel de pieza 3.6. La lesión no compromete las raíces dentarias ni desplaza estructuras (Fig. 13). La TC muestra una tumoración ósea radiolúcida, de alrededor de $2 \mathrm{~cm}$ de diámetro mayor, con adelgazamiento de la cortical interna y destrucción de la cortical externa con un trabeculado de aspecto desorganizado. Los contornos son lobulados, bien definidos, no compromete partes blandas adyacentes, comprimiendo el reborde superior de la mandíbula (Figs. 14,15 y 16).

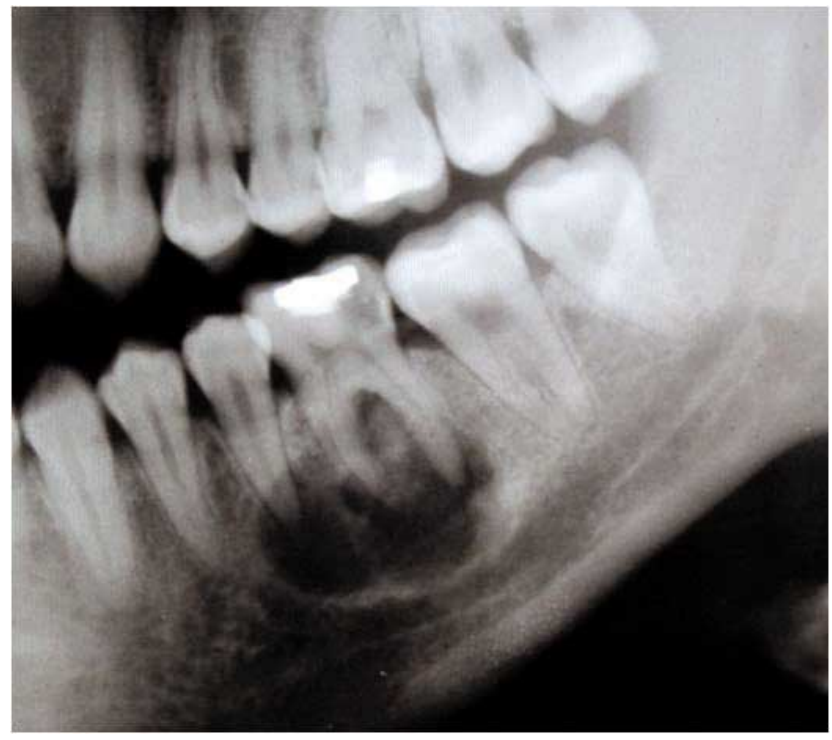

Fig. 13. Radiografía panorámica se observa imagen radiolúcida interradicular y periapical corticalizada con zonas radiopacas, de aspecto multilocular a nivel de pieza 3.6.

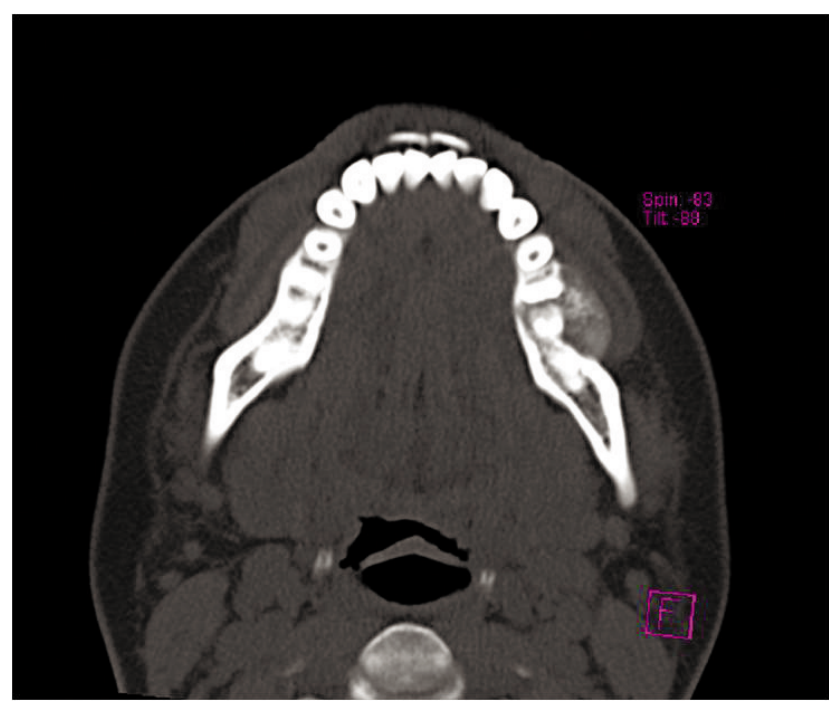

Fig. 14. TC mostrando una tumoración ósea radiolúcida, con adelgazamiento de la cortical interna y destrucción de la cortical externa con un trabeculado de aspecto desorganizado.

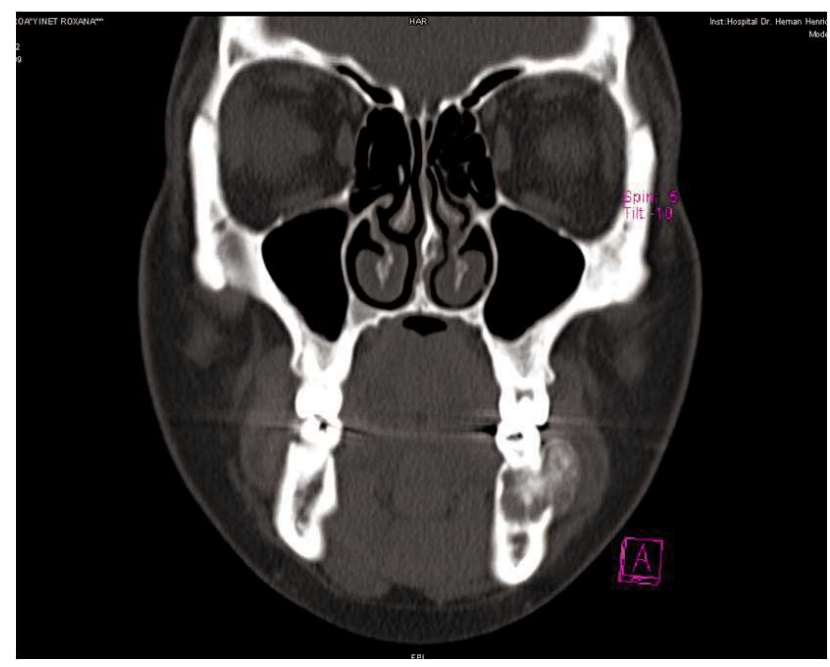

Fig. 15. TC muestra tumoración ósea radiolúcida, con adelgazamiento de la cortical interna y destrucción de la cortical externa con un trabeculado de aspecto desorganizado.

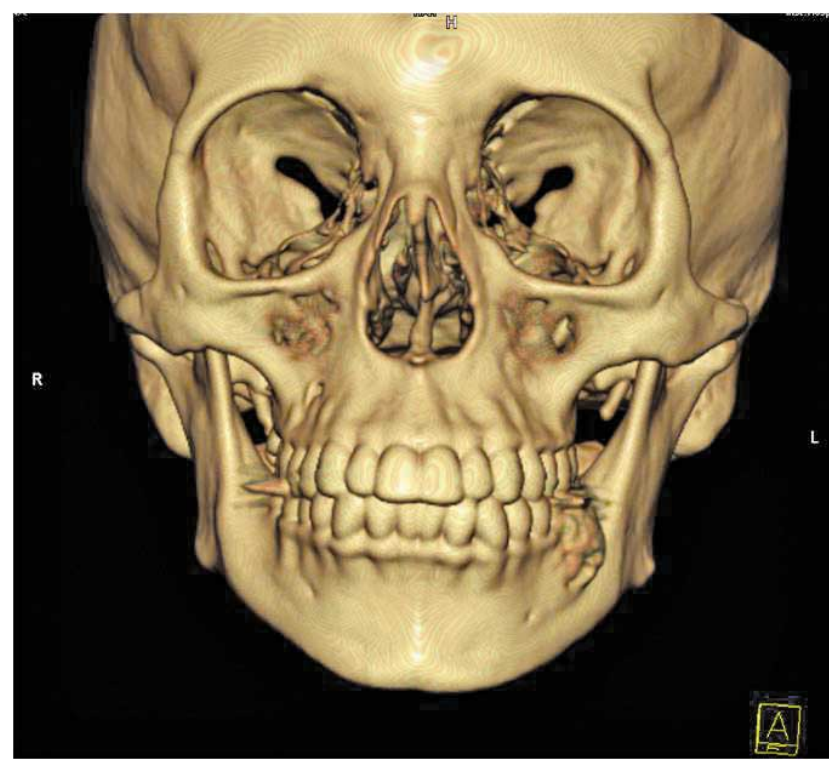

Fig. 16. TC de cabeza se observa tumoración ósea.

Bajo anestesia local, se realiza biopsia incisional de la lesión. En el estudio histopatológico es posible observar un tejido fibroso de celularidad moderada, presencia de células fusadas de núcleos normo típicos. Entre tejido fibroso existe presencia de tejido mineralizado de tamaño variable, compuesto por tejido esferoidal de tipo cemento, acelulares e hipocelulares. El resultado de este estudio arroja como diagnostico un fibroma cemento osificante (Fig. 17).

Bajo anestesia general se realiza abordaje intraoral y se procede a remover completamente la 


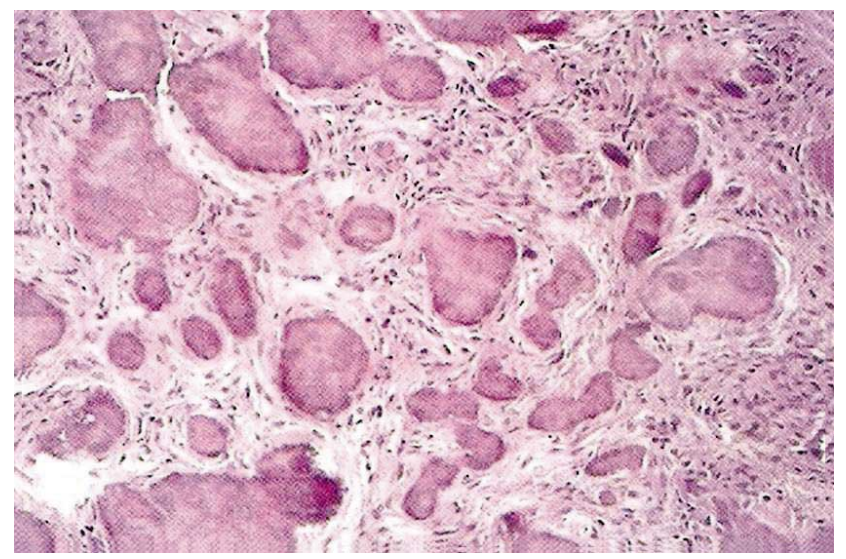

Fig. 17. Biopsia incisional de la lesión. Diagnóstico de fibroma cemento osificante

lesión a través de su plano de clivaje, junto con las piezas 3.5 y 3.6 (Fig. 18). La cavidad operatoria es rellenada con injerto óseo aloplástico ChronOs particulado $(0,5 \mathrm{cc})$ y se cierra herméticamente. No se presentaron complicaciones durante la cirugía. La pieza operatoria fue remitida a estudio histopatológico, el cual confirmo el diagnóstico de fibroma cemento osificante mandibular.

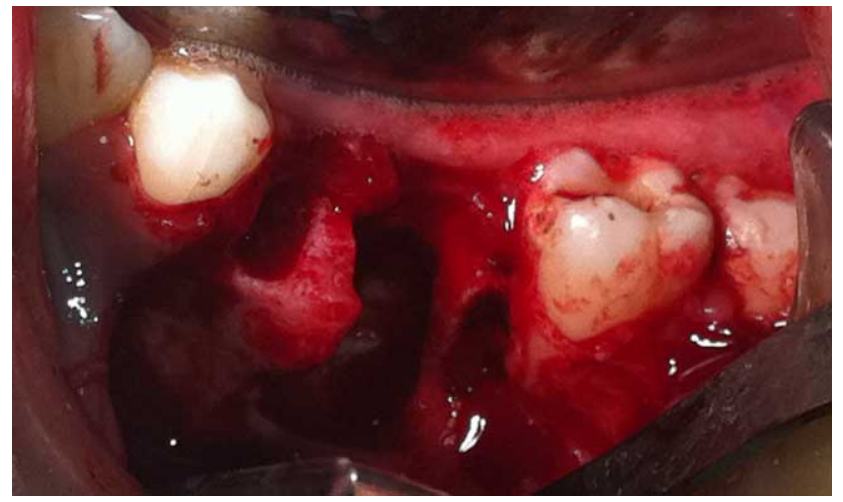

Fig. 18. Resultado remosión completa de la lesión junto con las piezas 3.5 y 3.6 .

La paciente evoluciono favorablemente, sin complicaciones y hasta la fecha no presenta signos de recidiva.

\section{DISCUSION}

Menzel fue el primero en describir una lesión fibro ósea en el año 1872. Estas lesiones benignas comparten características histopatológicas, como el reemplazo de un hueso normal por un tejido compuesto de fibras colágenas, fibroblastos y un grado variable de tejido mineralizado (Bertolini et al.).

Debido a sus características clínicas, radiológicas e histológicas, la clasificación de estas lesiones ha sido muy controversial (Domínguez \& Martín-Granizo). En el año 2005 la OMS modifico la clasificación de las lesiones maxilares, englobando al FCO dentro de los tumores de origen odontogénicos relacionados a tejido oseo, lo cual es aceptado en la actualidad (Waldrom, 1993; De Vicente et al., 1997).

Otra clasificación muy aceptada es la de Waldrom, quien divide estas lesiones en 3 grupos: del desarrollo, reactivas o displásicas, y neoplásicas (Waldron \& Giansanti, 1973). La etiología sigue siendo desconocida, aunque se postula que se origina por una estimulación de tipo traumática sobre células progenitoras, y este tipo de inducción provocaría un tipo de FCO más agresivo (Waldron \& Giansanti).

Los FCO más cercanos a la línea media facial, senos paranasales y maxilar presentan un comportamiento más agresivo que los de localización mandibular (Gunaseelan et al.). Otros autores relacionan la cantidad de cemento de la lesión con un comportamiento más agresivo (Hammer et al., 1968).

Se describe una variedad agresiva que se da en niños menores de 15 años y de preferencia en el maxilar. Su crecimiento es rápido y presenta una rica celularidad (Partridge et al., 2007). En este caso el tratamiento es remoción completa de la lesión mas curetaje. Posee una tasa de recidiva del $30 \%$ al $58 \%$ (Espinosa et al., 2006). Este subtipo también es controversial, ya que se dice que es un diagnostico subjetivo no basado en respaldo científico, ya que no todos se presentan en niños y no todos son agresivos (Lombardi et al.). Por lo tanto lo más importante es individualizar cada caso.

Más de la mitad de los pacientes con FCO desarrolla deformidad facial (Smith et al.). En un $85 \%$ de los casos la lesión se observa bien delimitada, de consistencia dura, de crecimiento lento, que desplaza los dientes, aunque estos conservan su vitalidad (Su et al.). Estudios indican que puede producirse divergencia radicular hasta en un $17 \%$ de los casos, mientras que la reabsorción radicular puede llegar hasta un $44 \%$ de los casos según distintos autores. El tamaño de la lesión también puede variar desde $0,2 \mathrm{~cm}$ hasta $15 \mathrm{~cm}$ (Pérez-García et al.). 
Existen muy pocos casos de fibromas cemento osificantes múltiples (Bertolini et al.). Regezzi \& Sciubba (1999) señalaron que los fibromas múltiples se presentan de manera muy esporádica, y que solo algunos pocos tienen un componente familiar. También señalaron que se han identificado translocaciones cromosómicas en algunos de los casos. Incluso, se han visto alteraciones cromosómicas en un pequeño número de FCO sometidos a estudio citogenético (Slootweg \& El-Mofty, 2005; Parham et al., 2004).

En la literatura existen solo dos reportes de casos de fibromas cemento osificantes múltiples que tenían un componente familiar (Yih et al., 1989; Canger et al.). Esto puede sugerir la posibilidad de la existencia de un componente hereditario en este tipo de lesiones (Smith et al.).

Es importante un adecuado diagnostico diferencial con la displasia fibrosa y la displasia ósea focal debido a que cambia el tratamiento de cada una de las lesiones (Young \& Rowson). La diferencia radica en que el FCO tiene un patrón de crecimiento centrífugo causando generalmente un borramiento de la basal mandibular, produciendo desplazamiento dentario y/o radicular (Canger et al.). Además, intra operatoriamente el FCO tiende a enuclearse intacto como una masa solida, debido a su capsula, lo cual es clave en su diferenciación. Otra diferencia radica en que en el FCO abundan los osteoclastos y osteoblastos, además que el patrón trabecular es más regular que en la displasia fibrosa, y contiene menos colágeno y elementos vasculares (Granados et al., 2006).

En la actualidad, el FCO continua siendo motivo de estudio y discusión. Esta patología requiere de un manejo multidisciplinario, y debe ser diagnosticada principalmente con la clínica, pero debe apoyarse de herramientas complementarias como la imagenología e histopatología para proporcionar un diagnóstico definitivo (Pérez-García et al.; Gunaseelan et al.).

VARGAS, F. I.; MAYER, O. C.; HERVOZO, S. P. \& NAVIA, G. E. Cemento-Ossifying Fibroma: Clinical, Radiological and Histological analysis of 2 cases in one family. Int. J. Odontostomat., 5(3):270-278, 2011.

ABSTRACT: The Cemento-ossifying fibroma (COF) is a benign fibro-osseous neoplasm of odontogenic origin, that is preferentially localized in the molar and premolar mandibular area. Our goal is to present cases of COF that occurred within the same family, describing clinical, radiological and histological characteristics, in addition to treatment and monitoring of each case.Two patients who are brothers, treated at the Hospital de Carabineros, who had mandibular COF, were included in this study. The complete evolution of each case is presented, highlighting the family component of this uncommon disease. All patients were treated with complete removal of the lesion and filling the bone defect with autologous iliac crest graft or synthetic particulated bone graft (ChronOS $\otimes$ ). One patient had recurrence of the injury and then presented a new COF on the contralateral side. In this type of injury it is very important to use all the diagnostic tools within our reach. The rarity of these lesions, and the great similarity of both clinical, radiological and histological features with other pathological conditions of the jaws, and a poorly conducted study can lead to wrong diagnosis and treatment affecting the quality of life of patients. The treatment of these lesions is conservative, and relapses are rare.

KEY WORDS: fibroma, benign neoplasm, bone graft, differential diagnosis.

\section{REFERENCIAS BIBLIOGRÁFICAS}

Bertolini, F.; Caradonna, L.; Bianchi, B. \& Sesenna, E. Multiple Ossifying Fibromas of the Jaws: A Case Report. J. Oral Maxillofac. Surg., 60:225-9, 2002.

Canger, E. M.; Celenk, P.; Kayipmaz, S.; Alkan, A. \& Gunhan, O. Familial ossifying fibromas: report of two cases. $J$. Oral Sci., 46:61-4, 2004.

De Vicente, J. C.; González, S.; Santamaría, J. \& Madrigal, B. Non odontogenic tumours of the jaws: classification, behavior and diagnosis. Med. Oral, 2:83-93, 1997.

Domínguez, L. \& Martín-Granizo, R. Análisis clínico, radiológico e histológico de los fibromas cemento- osificantes de los maxilares. Rev. Esp. Cirug. Oral y Maxilofac., 26:31-40, 2004.

Espinosa, S. A.; Villanueva, J.; Hampel, H. \& Reyes, D. Spontaneous regeneration after juvenile ossifying fibroma resection: a case report. Oral Surg. Oral Med. Oral Pathol. Oral Radiol. Endod., 102:e32-5, 2006.

Granados, R.; Carrillo, R.; Nájera, L.; García-Villanueva, M. \& Patron, M. Psammomatoid ossifying fibromas: Immunohistochemical analysis and differential diagnosis with psammomatous meningiomas of craniofacial bones. Oral Surg. Oral Med. Oral Pathol. Oral Radiol. Endod., 101:614-9, 2006. 
Gunaseelan, R.; Anantanarayanan, P.; Ravindramohan, E. \& Ranganathan, K. Large cemento-ossifying fibroma of the maxilla causing proptosis: a case report. Oral Surg. Oral Med. Oral Pathol. Oral Radiol. Endod., 104:e21-5, 2007.

Guzmán, J. E. \& Hernández, S. Fibroma osificante etmoidal, caso en paciente pediátrico. Acta Otorrinolaringol. Cir. Cabeza Cuello, 36:31-6, 2008.

Hammer, J. E. 3rd; Scofield, H. H. \& Cornyn, J. Benign fibroosseous jaw lesions of periodontal membrane origin: an analysis of 249 cases. Cancer, 22:861-78, 1968.

Kaplan, I.; Manor, I.; Yahalom, R. \& Hirshberg, A. Central giant cell granuloma associated with central ossifying fibroma of the jaws: a clinicopathologic study. Oral Surg. Oral Med. Oral Pathol. Oral Radiol. Endod., 103:e35-41, 2007 .

Lello, G. E. \& Sparrow, O. C. Craniofacial poliostotic fibrous displasia. J. Maxilofacial. Surg., 13:267-72, 1985.

Lombardi, A.; Cerullo, M.; Garzón, J. C.; Martínez, J. \& Cuella, E. Fibroma osificante de mandíbula: Presentación de un caso y revisión de la literatura. Rev. Esp. Cirug. Oral y Maxilofac., 31:242-8, 2009.

Manganaro, A. M.; Ragno, J. R. Jr. \& Karlis, V. Mixed radiolucent/radiopaque lesion of the mandible. J. Oral Maxillofac. Surg., 55:1456-9, 1997.

Parham, D. M.; Bridge, J. A.; Lukacs, J. L.; Ding, Y.; Tryka, A. F. \& Sawyer, J. R. Cytogenetic distinction among benign fibro-osseous lesions of bone in children and adolescents: Value of karyotypic findings in differential diagnosis. Pediatr. Dev. Pathol., 7:148-58, 2004.

Partridge, J. E.; Hicks, M. J. \& Marchena, J. A 7-Year-Old with Progressive Mandibular Expansion. J. Oral Maxillofac. Surg., 65:2047-55, 2007.

Pérez-García, S.; Berini-Aytés, L. \& Gay-Escoda,C. Fibroma osificante maxilar: Presentación de un caso y revisión de la literatura. Med. Oral, 9:333-9, 2004.

Regezzi, J. A. \& Sciubba, J. J. Oral pathology. 3rd ed. Philadelphia, W. B. Saunders, 1999. pp.357-60.

Sakode, S.; Shiba, R. \& Irino, S. Immediate reconstruction of the mandible in a patient with ossifying fibroma by replantation of the resected segment after freezing. $J$. Oral Maxillofac. Surg., 50:521-4, 1992.

Smith, S. F.; Newman, L.; Walker, D. M. \& Papadopoulos, H. Juvenile Aggressive Psammomatoid Ossifying Fibroma: An Interesting, Challenging, and Unusual Case Report and Review of the Literature. J. Oral Maxillofac. Surg., 67:200-6, 2009
Slootweg, P. J. \& El-Mofty, S. K. Ossifying fibroma. In: Barnes, L.; Eveson, J. W. \& Reichart, P. (eds). Pathology and Genetics of Head and Neck Tumors. World Health Organization Classification of Tumors. Lyon, IARC Press, 2005. pp.319-22.

Su, L.; Weathers, D. R. \& Waldrom, C. A. Distinguishing features of focal cemento-osseous dysplasias and cemento-ossifying fibromas. A pathologic spectrum of 316 cases. Oral Surg. Oral Med. Oral Pathol. Oral Radiol. Endod., 84:301-9, 1997.

Waldrom, C. A. Fibro-osseous lesions of the jaws. J. Oral Maxillofac. Surg., 51:828-35, 1993.

Waldron, C. A. \& Giansanti, J. S. Benign fibro-osseous lesions of the jaws: a clinical-radiologic-histologic review of sixtyfive cases. Part II Benign fibro-ossoeus lesions of periodontal ligament origin. Oral Surg. Oral Med. Oral Pathol., 35:340-50, 1973.

Yih, W. Y.; Pederson, G. T. \& Bartley, M. H. Jr. Multiple familial ossifying fibromas: relationship to other osseous lesions of the jaws. Oral Surg. Oral Med. Oral Pathol., 68:754-8, 1989.

Young, N. \& Rowson, J. E. Cementifying fibroma of the frontal bone: A case report. B. J. Oral Maxillofac. Surg., 45:667-9, 2007.

Dirección para correspondencia:

Ilich Vargas F.

Cirujano Maxilofacial

Hospital Mutual de Seguridad

Santiago

CHILE

Email: cristopher.mayer@gmail.com

Recibido : 15-10-2011

Aceptado: 08-11-2011 